\title{
Assessing time knowledge in children aged 10 to 11 years
}

\author{
Nicola Brace (iD) ${ }^{1}$, Clare Doran², Janet Pembery ${ }^{3}$, Emma Fitzpatrick ${ }^{1}$, \\ Rosalind Herman (D) 1,*
}

${ }^{1}$ City, University of London, School of Health Sciences, Division of Language and Communication Science UK ${ }^{2}$ Bedfordshire Community Health Service, UK

${ }^{3}$ Central and North West London NHS Foundation Trust, UK

\section{ARTICLE HISTORY}

Received: 01 May 2019

Revised: 13 Septembe 2019

Accepted: 26 October 2019

\section{KEYWORDS}

Time knowledge,

Assessment,

Clock reading,

Time orientation,

Estimating time intervals

\begin{abstract}
The acquisition of time knowledge involves learning how to read clocks, estimate time, read dates and learn about temporal sequences. Evidence suggests that many of these competencies are acquired by 10 years of age although not all children may follow this developmental path. The main purpose of this study was to collect normative data for a screening tool that assesses time knowledge. These data identify the prevalence and pattern of difficulties with time knowledge among a UK sample of Year 6 pupils (aged 10 to 11 years). The Time Screening Assessment tool (Doran, Dutt \& Pembery, 2015), designed to assess time knowledge, was administered individually to a sample of 79 children. Findings revealed a median overall score of 32 out of a maximum score of $36.25 \%$ of children performed at or close to ceiling, however seven children scored more than -1.5 standard deviations below the mean. The value of these findings to practitioners working with children in schools is discussed.
\end{abstract}

\section{INTRODUCTION}

In everyday life, keeping track of time allows us to organise activities and coordinate these with others, and is a skill that is acquired during childhood. Burny, Valcke and Desoete (2009) suggested that what develops is a range of time-related competencies including the accurate reading of clocks and calendars, and the ability to use mental timelines to measure and estimate time intervals. Furthermore, Burny et al. highlighted that the specific skill of reading clocks draws on a number of sub-competencies including language skills, memory, numeracy and spatial abilities. They explained that as well as being able to count and have a basic understanding of fractions, children need to learn to express correctly the relationship between the hour and the minute. For the relative expression 'ten past eleven' the minute is mentioned before the hour; however, for the absolute expression 'eleven ten' the hour comes first.

Clock reading has been explored in a number of studies. Siegler and McGilly (1989) concluded from North American studies conducted in the 1970s and 1980s that children develop the ability to tell the time from analogue clocks in a particular sequence. By 6 years of age many can tell

CONTACT: Rosalind Herman $\bigotimes$ R.C.Herman@city.ac.uk Đ Division of Language and Communication Science, School of Health Sciences, City, University of London, Northampton Square, EC1V 0HB 
whole-hour times, by 7 or 8 years 5-minute times, and between the ages of 8 and 10 years many could tell 1-minute times. However, reading the time from digital clocks does not follow the same pattern. A study by Friedman and Laycock (1989) involved participants from five age groups, with 32 North American children in each (mean ages: 6;6, 7;7, 8;6, 9;7 and 10;6). Most in the youngest group could tell whole-hour, half-hour and 1-minute times when reading a digital clock, and performance was near perfect in the second age group. However, their performance when reading analogue clocks varied according to the time being displayed. Whole-hour readings were accurate in the youngest age group and half-hour times in the second age group, but more complex 1-minute times (such as 2:43) remained difficult for at least some children in the oldest age group. This early research suggests that analogue clock reading is a complex skill acquired over a period of time, but in the most part it is achieved by 10 years of age.

Several studies have shown that numerical skills may affect the acquisition of clock reading skills. Andersson (2008) compared clock reading in 182 children attending school in Sweden with a mean age of 125 months. Those with mathematics difficulties were found to have substantial problems with reading both analogue and digital clocks. Burny, Valcke and Desoete (2012) sampled 725 children from eight Belgian primary schools and identified 154 children with mathematics difficulties who performed worse than the others on clock reading tasks; furthermore, telling the time accurately to 1-minute and 5-minutes was difficult with both analogue and digital clocks. Analysis of errors suggested both miscounting and misinterpreting, with the latter most likely due to a combination of difficulties, including poorer counting strategies and absent memory representations. For example, reporting 10:04 rather than 10:20 suggests that the child was not counting in fives, reflecting a lack of knowledge that the ' 4 ' on the analogue clock means ' 20 '.

Clock reading is just one aspect of time knowledge. Other research has focused on the acquisition of knowledge about temporal sequences. In a US study, Friedman (1991) looked at children's ability to date events on a time scale. The children were aged 4, 6 and 8 years with 14 children in each age group. They were asked about two events they had experienced, one staged seven weeks and the other one week prior to testing. The youngest group could accurately decide which of the two events was more recent, and therefore had a sense of different times in the past, but it was only the 6 and the 8-year old children who could estimate when the older event occurred and who showed awareness of day, month and season. In a further study, Friedman (1992) compared the same three age groups, asking children to recall events from specific points during the previous year and, whilst performance improved with age, only $56 \%$ of those in the oldest group could position these multiple events into their correct temporal order. These findings suggest that acquisition of time-awareness continues beyond the age of 8 years. Based on these and other findings, Friedman (2005) proposed that children first learn the order of the days of the week and months of the year, using a list-based representation. As they grow older they begin to form representations of longer time scales such that by 10 years of age they have a sense of the annual cycle, for example they can judge how long it is until next summer, and become aware of temporal distances between the days of the week or months of the year, for example that April and October are quite far apart.

Another component of time knowledge concerns the ability to judge how long something takes. A study by Quartier, Zimmermann and Nashat (2010) compared Swiss French-speaking children aged between 6 and 13 years, 22 with attention-deficit/hyperactivity disorder (ADHD) and 22 controls. They found that children with ADHD who were younger than 10 years of age had more difficulty than controls with conventional time concepts such as dates, durations and order of events. Although those older than 10 years of age showed conventional time knowledge, they differed significantly from the control group in terms of their ability to organise time, for example to plan forward and meet deadlines. Children with autism spectrum 
condition (ASC) have also displayed difficulties with time-based judgements (e.g. Williams, Boucher, Lind \& Jarrold, 2013).

A number of researchers have developed questionnaires to measure the different components of time knowledge. Quartier et al. (2010) used the Time Concept Questionnaire (Quartier, 2008) consisting of questions relating to time orientation, conventional time sequences, objective durations, subjective durations and anticipation. Labrell, Mikaeloff, Perdry and Dellatolas (2016) developed their own Time Knowledge Questionnaire which included four subtests: time orientation (e.g. 'What day is it today?), sequences in relation to months and seasons, time units (e.g. 'Is a minute shorter or longer than a second?') and telling the time on a clock. There were three other subtests designed to measure understanding of the lifespan, the child's own birthday and time estimation. The latter was assessed through a question about the duration of the interview. They administered this to 105 French children from state schools, ranging in ages from 6 to 11 years, and found that although time knowledge increased with age, different subtests revealed different patterns. For example, time orientation was at ceiling from 7 years of age, whereas time estimation continued to improve between 9 and 10 years of age. Furthermore, when controlling for age they found significant correlations between some subtests but not all and suggested that what they were measuring might not be unidimensional. Whereas Labrell et al. (2016) were concerned with the development of time knowledge between the ages of 6 to 11 years, Dutt and Doran (2013) reported data using a similar questionnaire from 20 young people, aged 13 to 17 years, who had been referred for assessment or therapy to a Youth Offending Team. They found nine young people had difficulties associated with estimating and telling the time, with calendar time (i.e. naming the months in the correct order and interpreting a short date), and with understanding the word 'fortnight'. Importantly, these findings are in contrast to the research evidence presented so far which suggested that time knowledge competencies are acquired fully by around 10 years.

The questionnaire used in the Dutt and Doran study has since been published along with a resource pack (Doran, Dutt and Pembery, 2015). It is called the Time Screening Assessment and was developed because of the authors' experiences as Speech and Language Therapists. As therapists they found some young people to have a poor sense of time, they were either missing appointments or were late, and had difficulties with temporal sequences and clock reading. Colleagues working in secondary schools had highlighted similar difficulties among some pupils aged older than 10 years. The Time Screening Assessment was developed as a tool to allow the identification of children who are not acquiring time knowledge according to the usual developmental trajectory and assesses knowledge that is taught in schools in England before the age of 10 years. According to the UK National Curriculum Statutory Guidance (2013) it is a statutory requirement for pupils to have been taught by nine years of age clock reading skills (including from analogue clocks), temporal sequences (identifying chronological order using language, and recognising and using language related to dates) and also estimating time and comparing the durations of events.

The aim of the present study was to explore the incidence of poor time knowledge in a nonclinical group of children aged 10 to 11 years and thereby provide normative data for the Time Screening Assessment. Based on the findings of previous research, suggesting that time-related competencies are achieved by 10 years of age, the majority of scores were predicted to be at ceiling, and based on Dutt and Doran (2013) it was also predicted that some participants might not score highly on this assessment tool. No predictions were made with regards to the possible effects of gender, type of school or age, however these were explored when analysing overall performance. Based on the findings of Labrell et al. (2016), it was also predicted that there would be significant correlations between different sections of the questionnaire. 


\section{METHOD}

\subsection{Design of the study}

This study used a questionnaire designed to measure time knowledge.

\subsection{Participants}

This study received ethical approval from City, University of London. In order to obtain a sample of 10 to 11-year olds representative of those attending state schools in South East England, five different schools were approached that were located in Buckinghamshire and Greater London. Participants were recruited from Year 6 of five government-funded primary schools, two of which were located in a village (with 30 and 88 pupils), two in a town (with 45 and 60 pupils) and one in a large city (with 90 pupils). At village and town schools the proportion of pupils for whom the school received a pupil premium (additional funding for disadvantaged children) was below average, as was the proportion of pupils who had special educational needs. The city school had an above average proportion of pupils receiving the pupil premium, with special educational needs, and with English as an additional language.

All year 6 pupils at each school took home information about the study. In compliance with ethics approval, parents were invited to return a signed consent form to the Year 6 teacher. Parents were also asked to provide optional information, including their child's date of birth (in order to accurately score one question), whether their child had received a diagnosis of $\mathrm{ADHD} / \mathrm{ASC}$, and whether they had received any speech and language therapy.

Of the 81 children for whom a consent form was returned 37 were girls and 44 were boys. They were aged between 123 and 136 months (mean age $=129.74$ months, SD = 3.19). The final sample included in data analysis comprised 32 pupils from village schools, 34 from town schools and 13 from a city school. The criteria for including data in the analysis were that participants did not have a diagnosis of ADHD or ASC as both conditions have been linked to difficulties with time-based judgements. Two children (boys) did not fit the inclusion criteria and their responses were excluded from data analysis.

\subsection{Materials}

The Time Screening Assessment (Doran et al., 2015) has five sections, with multiple questions in each: Calendar time; Clock time; Time vocabulary; Organisation of time; and Estimation of time. In total, there are 25 questions, with the majority requiring a response that is either correct or incorrect (e.g. What does 'fortnight' mean? In which month is Christmas? What is the time shown here?). Four questions ask respondents to indicate a strategy (e.g. How do you know when it is time to get up in the morning?) and four questions ask for an estimate of time duration (e.g. Approximately how many minutes does a song on the radio and a school lesson last?).

Following the advice of the authors of this measure, and based on their experience of using the tool, three questions were amended to suit the age group and diversity of cultures of the participants: 'Explain exactly what each number means in this date' was amended to 'What date is this?'; 'How long do you think this assessment has taken?' was amended to 'How long has it taken to answer these questions'; and 'Which season is usually hot?' was amended to 'Which season is usually hot here in England?'.

Three images were printed on A4 paper for the purposes of asking three of the questions: a date in a short format (03/06/12); a digital clock showing 7:20; and an analogue clock showing 11:05. The validity and reliability of the assessment tool has not to date been evaluated, although it does have face validity as it assesses time knowledge taught according to the UK National Curriculum Statutory Guidance. Investigation of scorer reliability was carried out as part of this study. 


\subsection{Procedure}

All participants were assessed individually in school by the primary author. Children were invited to sit at a quiet desk outside of their classroom. An analogue clock was placed on the desk. Each question from the tool was read out and their response was recorded in writing, either verbatim or précised. Positive encouragement was provided throughout, regardless of whether responses were correct, and assessments lasted between 10 and 15 minutes. At the end of the session children were asked if they found any of the questions difficult. Responses to this final question were not scored or included in the analysis, but reassurance was provided if any concerns were raised.

\subsection{Scoring}

Scoring followed the guidance provided by Doran et al. (2015). Four questions in Calendar time were coded 0 (incorrect) or 1 (correct). In terms of providing today's date, participants only had to specify the correct day and month, and for the date of their birthday they could be prompted to provide the year. When asked to name the seasons 1 point was awarded for naming all four seasons and a further point for naming them in the correct order. When asked to explain a short date 1 point was awarded for correct naming of each of the day, month and year. When asked to name the months of the year in order 3 points were awarded if all 12 months were provided in the correct order, 2 points if one or two errors of omission or order, 1 point if three errors and 0 points if four or more errors.

Three questions in Clock time were coded 0,1 or 2 points for each clock shown (digital and analogue), with 2 points being were awarded if the time was correctly described using both relative and absolute expressions, 1 point if one of these expressions was used and 0 if the time was not correctly identified. No points were awarded for answers such as ' 50 past 7' or ' 35 past 11 '. When asked what each clock would be in half an hour, 2 points were awarded if the correct time was provided for both clocks and 1 point if correct for one clock. For the final question 'What is the time now?', responses were given 1 point if correct to within two minutes. The four questions in Time vocabulary were coded as either 0 (incorrect) or 1 (correct), as were the four questions in Organisation of time where 0 was given if the response indicated that the child predominantly relied on another person and 1 if the child used a strategy that did not involve another person.

For Estimation of time, three questions were coded as 0,1 or 2 points. Each involved asking children to estimate how long two activities lasted (minutes, hours or weeks/months). 1 point was awarded if children estimated the length of a song as 2-5 minutes, 1 point for correct length of a lesson, 1 point for saying the length of a film was between 11/4-3 hours, 1 point for saying the school day was 6-8 hours, 1 point for estimating the length of a term as 12-14 weeks (or half term 6-8 weeks) and 1 point for saying the length of the school summer holiday break was 5-7 weeks. The final three questions in this section were coded as either 0 or 1 point. Reasonable answers to name something that takes an hour to do were scored as 1 point, for example football practice or English homework. Responses when asked to estimate how many months or weeks until their next birthday were given 1 point if correct to within a month. Responses when asked how long the assessment had taken were awarded 1 point if correct to within five minutes.

\subsection{Reliability}

A sample of 30 assessments was independently scored by a second person who was briefed on the scoring system outlined above. An intra-class correlation coefficient (ICC) was used to assess reliability of the overall score given by each scorer. The reliability coefficient was calculated as .981 , with $95 \%$ CI $(.950, .992)$ indicating a high level of agreement between the two scorers. 


\section{RESULTS}

\subsection{Overall performance on the Time Screening Assessment}

The scores for each question for 79 child participants were analysed and an overall score, out of a maximum of 36, was calculated for each participant. The distribution of overall scores, shown in Figure 1, is negatively skewed.

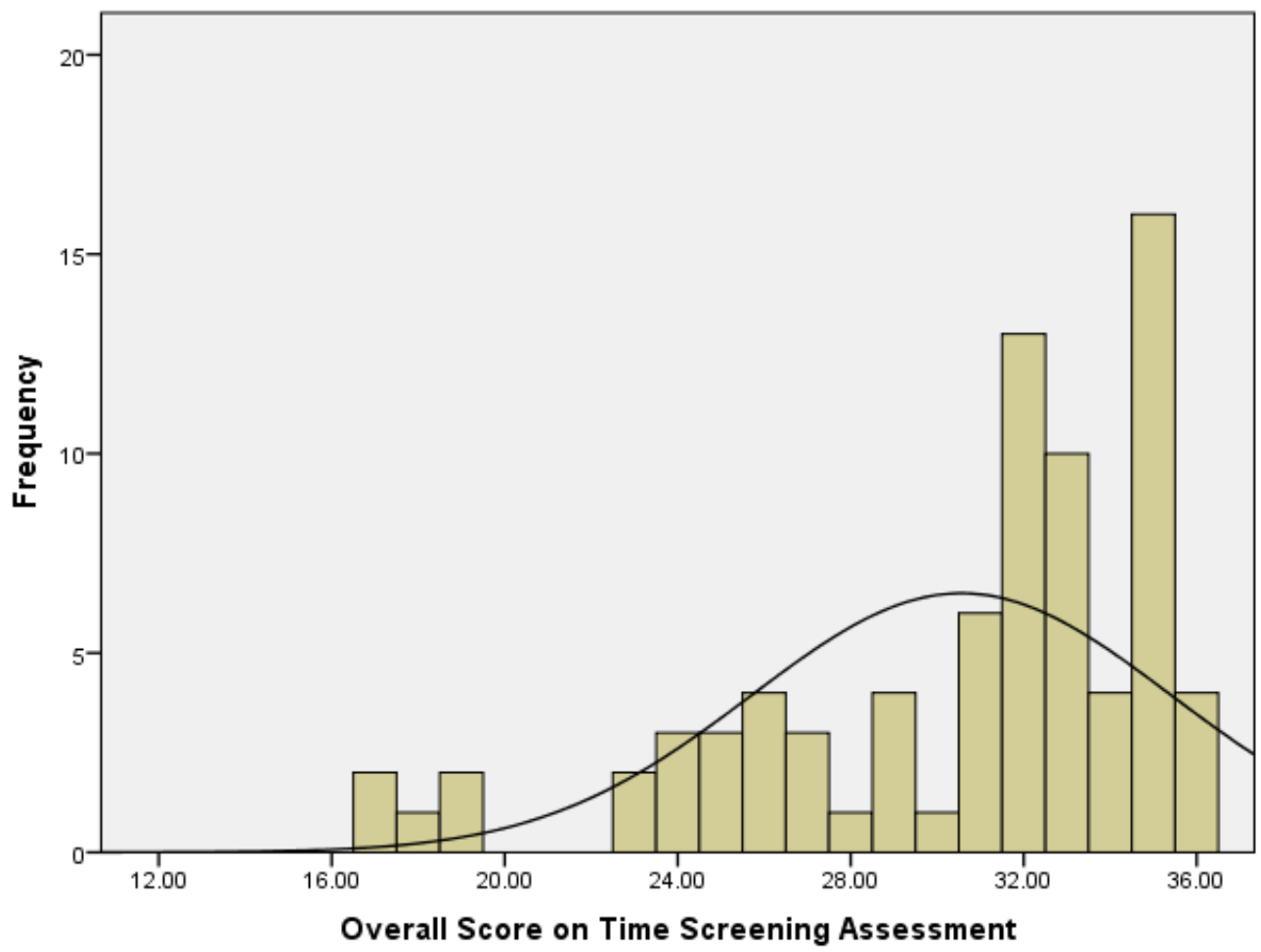

Figure 1. Distribution of overall scores on the Time Screening Assessment

$25.4 \%$ of the sample scored full (36/36) or almost full marks (35/36). A further $43.2 \%$ scored between 30/36 and 34/36. However, 31.6\% scored below 30/36. Transformation to $\mathrm{z}$ scores revealed that $16.5 \%$ were more than -1 standard deviation from the mean, with an overall score lower than $26 / 36$. Seven scores were in excess of -1.5 standard deviations below the mean, with five scores in excess of -2 standard deviations.

Table 1. Descriptive statistics for overall scores on the Time Screening Assessment, broken down by gender

\begin{tabular}{lllllll}
\hline Gender & $N$ & Mean & Median & SD & Minimum & Maximum \\
\hline Boy & 42 & 30.52 & 32 & 5.19 & 17 & 36 \\
\hline Girl & 37 & 30.59 & 32 & 4.50 & 19 & 36 \\
\hline Overall & 79 & 30.56 & 32 & 4.85 & 17 & 36 \\
\hline
\end{tabular}

Table 1 suggests that as a group there was a range in the scores achieved although the majority were towards the top end. The performance of boys overall appears similar to that of the group of girls, and a Mann-Whitney $U$ test confirmed that there was no significant difference $(U=$ 775.50, $N_{1}=42, N_{2}=37, p=.988$, two-tailed). 
Table 2. Descriptive statistics for overall scores on the Time Screening Assessment, broken down by type of school

\begin{tabular}{l|llllll}
\hline $\begin{array}{l}\text { Type of } \\
\text { school }\end{array}$ & $N$ & Mean & Median & SD & Minimum & Maximum \\
\hline Village & 32 & 32.28 & 33 & 3.63 & 18 & 36 \\
\hline Town & 34 & 30.44 & 32 & 5.37 & 17 & 36 \\
\hline City & 13 & 26.61 & 25 & 3.85 & 19 & 32 \\
\hline
\end{tabular}

Table 2 above suggests that overall the scores of the children attending the school located in a large city were lower than those of the children attending the schools located in the village or town. A Mann-Whitney U test was used to perform three pairwise comparisons (Bonferronicorrected $p$-value $=.017)$. No statistically significant difference emerged when comparing village and town schools $\left(U=453.00, N_{1}=32, N_{2}=34, p=.237\right.$, two-tailed), however the scores for the city school were significantly lower than those from the town schools $(U=$ $100.00, N_{1}=34, N_{2}=13, p<.005$, two-tailed) and those from the village schools $(U=42.50$, $N_{1}=32, N_{2}=13, p<.001$, two-tailed). It is worth nothing, however, that of the seven lowest scoring participants, one came from one of the village schools, four from the town schools (two from each) and two from the city school.

As there was a range in age from 10 years and 3 months to 11 years and 4 months, Spearman's $r$ was calculated to explore any relationship between age and overall assessment score. There was a significant positive correlation between age and the overall score $\left(r_{s}=.229, N=79, p<\right.$ .05 , two-tailed), however the strength of the correlation is weak and only $5.24 \%$ of the proportion of the variation in the overall scores is explained by age.

\subsection{Performance in each section of the Time Screening Assessment}

In addition to calculating an overall score, a score for each section of the Time Screening Assessment was calculated for each child.

Table 3. Descriptive statistics for each section of the Time Screening Assessment

\begin{tabular}{llllll}
\hline $\begin{array}{l}\text { Section (maximum possible } \\
\text { score) }\end{array}$ & Mean & Median & SD & Minimum & Maximum \\
\hline Calendar time (12) & 10.56 & 11 & 1.93 & 3 & 12 \\
\hline Clock time (7) & 5.84 & 7 & 1.85 & 1 & 7 \\
\hline Time vocabulary (4) & 3.06 & 3 & 0.88 & 1 & 4 \\
\hline Organisation of time (4) & 3.18 & 3 & 0.87 & 1 & 4 \\
\hline Estimation of time (9) & 7.92 & 8 & 1.22 & 4 & 9 \\
\hline
\end{tabular}

Table 3 shows the median score to be close to or at the maximum possible, suggesting that the majority of children provided accurate responses to each section. Of the seven lowest-scoring participants, all scored below the median in Estimation of time and Calendar time and, except for one participant, Clock time scores were low. In contrast, all but two of the seven achieved the median in the section Organisation of time.

In relation to Calendar time, nearly half the sample achieved the maximum score. While all children knew their own birthday, $10 \%$ responded incorrectly when asked what today's date was and about $35 \%$ could not identify the day/month/year when shown a short date. Many misidentified the month '06' as July and some did not recognize '12' as being '2012'. Just over 
a quarter did not name all the months of the year in the correct order and just over a quarter did not name all four seasons in the correct order.

In terms of Clock time, 63\% achieved the maximum score, and a further 10\% scored 6/7. Approximately one quarter scored $4 / 7$ or less. Whereas all children were able to read correctly the time displayed by an image of a digital clock, using the relative expression 'twenty past seven' and/or the absolute expression 'seven twenty', 11 participants (nearly 14\%) did not read the time when shown an image of an analogue clock using either type of expression. Furthermore, whereas only 10 children did not say accurately what the time would be in half an hour when looking at the digital clock, 25 children did not provide a correct response when shown the image of the analogue clock. Finally, 10 children did not provide an accurate response when asked the time.

For the Time vocabulary questions, almost $40 \%$ achieved the maximum score. Almost half could not define the word 'fortnight' correctly and just over a third did not know the meaning of the word 'century'. When asked about Organization of time, $43 \%$ achieved the maximum score. The remainder responded that they were reliant on another person in relation to one of four scenarios: knowing when it is time to get up in the morning; knowing when it is time to leave for school; how they remembered an important date and an important time.

Finally, $40 \%$ achieved the maximum score with Estimates of time durations. Over $90 \%$ were able to correctly answer how many minutes a song and a school lesson lasts, and how many hours a film and a school day lasts, and all but one could name something that takes about an hour to do. However, $28 \%$ were unable to correctly estimate how many weeks a school term lasts and $15 \%$ did not know how many weeks the school summer holiday lasts. Furthermore, $16.5 \%$ children could not correctly estimate how long until their next birthday, and $21.5 \%$ did not estimate correctly how long the assessment session had lasted.

To explore whether there were significant relationships between the section scores, a series of Spearman's r were calculated. There were significant relationships between most of the section scores, with the exception of Organisation of time. The results are presented in Table 4.

Table 4. Correlations $\left(r_{s}\right)$ between Time Screening Assessment section scores

\begin{tabular}{lllll}
\hline$N=79$ & Clock Time & $\begin{array}{l}\text { Time } \\
\text { vocabulary }\end{array}$ & $\begin{array}{l}\text { Estimation of } \\
\text { time }\end{array}$ & $\begin{array}{l}\text { Organisation of } \\
\text { time }\end{array}$ \\
\hline Calendar time & $.457^{*}$ & $.475^{*}$ & $.445^{*}$ & .104 \\
\hline Clock time & & $.495^{*}$ & $.342^{*}$ & .254 \\
\hline Time vocabulary & & $.452^{*}$ & .091 \\
\hline Estimation of time & & & & -.027 \\
\hline
\end{tabular}

$* p<.005$ (the Bonferroni-corrected $p$-value for performing ten correlations)

\section{DISCUSSION and CONCLUSION}

The primary aim of this study was to use the Time Screening Assessment (Doran et al., 2015) to explore difficulties with time knowledge in children aged 10 to 11. Among 79 Year 6 pupils approximately $25 \%$ of children performed at or close to ceiling and a further $43 \%$ achieved at least 30/36, with $32 \%$ scoring less that 30/36. The large proportion of children achieving high scores on this assessment supports previous research which suggests that by 10 years of age children have acquired the skills of reading clocks (e.g. Freidman \& Laycock, 1989) and knowledge about temporal sequences, such as the months of the year (e.g. Friedman, 1992). However, the distribution of scores showed a long tail of scores lower than 30/36 and transformation to $\mathrm{z}$ scores indicated that the performance of $16.5 \%$ of the sample was in excess 
of -1 standard deviation from the mean, including seven cases that were more than -1.5 standard deviations away from the mean.

This finding supports the observation of Dutt and Doran (2013) that some young people have poor time knowledge. However, they identified a larger proportion with difficulties, namely nine out of 20 young people aged 13-17, and the disparity in prevalence is likely to reflect differences between the samples. The sample in the Dutt and Doran study comprised young people referred to the Youth Offending Team, and there is evidence showing that a high proportion of youth offenders have language and communication difficulties (e.g. Bryan, Freer \& Furlong, 2007).

Poor numerical skills have been found to hamper the acquisition of clock reading skills (e.g. Burny et al., 2012), and when examining the data from the lowest scoring participants, six of the seven cases showed a particularly low score in the section assessing clock reading. However, clock reading was not the only competency impaired among these participants as scores were also low in the sections assessing temporal sequences and time estimation. The finding that performance was poor in sections other than clock reading is consistent with that reported by Dutt and Doran (2013), who also observed difficulties with the order of the months and estimating time in addition to clock reading problems.

There was no significant effect of gender, and only a weak significant correlation was observed between age and overall score achieved on the Time Screening Assessment, with age accounting for around 5\% of the variation in the overall scores. This small effect of age may reflect that certain aspects of time knowledge continue to develop beyond 10 years of age. Labrell et al. (2016) looked at the development in time knowledge from age 6 to 10 years and found that judging interview duration continued to improve after age 9. In the present study, $21.5 \%$ did not accurately estimate the length of the assessment.

Statistically significant correlations were observed between most, but not all, of the sections of the Time Screening Assessment, in line with the findings of Labrell et al. (2016). That clock reading abilities might correlate with other aspects of time knowledge is consistent with the point made by Burny et al. (2009), namely that clock reading draws on a number of subcompetencies, including memory, numerical, spatial and language skills. The overall scores from the section Organisation of time, designed to assess reliance on others for time organisation, did not correlate with those from the other sections, and this might reflect the fact that children can acquire good time knowledge but nevertheless continue to rely on another to be on time. Alternatively, children may develop strategies to be on time, for example using their phone as an alarm and for reminders, without acquiring a solid knowledge base concerning dates and temporal sequences, or the ability to estimate the duration of events accurately.

When exploring the pattern of errors that children were making, it is worth highlighting that approximately $14 \%$ of the children sampled in the present study were not able to accurately read the time shown on the image of an analogue clock, and approximately 13\% could not tell the time from a real analogue clock, whereas all were able to read the time displayed on an image of a digital clock. This pattern is consistent with the findings from Friedman and Laycock (1989) who found that although performance reading a digital clock was near perfect before 10 years of age, reading an analogue clock depended on the time being displayed, with whole-hour and half-hour times being easier, and that even the oldest age group of 10 to 11-year olds had difficulty with more complex time such as 2:43. Several studies have established that numerical skills are implicated in accurate clock reading from both analogue and digital clocks (Andersson, 2008; Burny et al., 2012), and the poor performance observed here may in part be attributed to weak numerical skills. As these were not assessed in the present study it was not possible to explore their contribution further. 
It is also worth noting that although performance in identifying the day of the week was close to ceiling (when asked about the day after tomorrow and what day it was two days ago), approximately a quarter of the children sampled in the present study were not able to identify all the months of the year in the correct order and about a quarter could not name the four seasons in the correct order. This finding contrasts with that of Friedman (1992) who found that children aged 8 to 9 years showed awareness of the months and seasons and could order the seasons. However, Friedman (2005) suggested that acquisition of time-awareness continues beyond the age of 9 years when children begin to form representations of longer time scales. The findings of the present study suggest that in this sample children had good representations for the relatively short time scale of a week, but that for some children representations of longer time scales were still developing. Consistent with this explanation is the pattern of errors observed in relation to estimating time durations. Performance overall was poorer when children were asked about events of longer durations, such as how many weeks a term lasted compared to shorter durations such as how many minutes a song lasted.

There are two issues to consider when evaluating the contribution of the present study. Firstly, the sample comprised children whose parents actively consented to their participation and therefore may not be representative as it has been argued that the opt-in (active) consent process may result in a reduced sample size and an increased possibility of sampling bias, limiting the validity and generalizability of the study results (see Hollmann \& McNamara, 1999). In the present study, there was a low response rate for each of the five schools; the lowest was for the city school with only $14 \%$ of parents returning consent forms and the highest was from one town school with a return rate of almost $38 \%$. Also, the sample was limited to one region of England and therefore the findings reported here may not generalize to other regions of England or other countries.

Secondly, at the present time, there is limited data concerning the reliability and validity of the Time Screening Assessment. In the present study, there was a high level of agreement between the two people scoring the responses from 30 children in the present study pointing to interrater reliability. Furthermore, there were correlations between most sections of the tool indicative of internal consistency. Further research assessing test-retest reliability is necessary. In terms of validity, the Time Screening Assessment has face validity as it assesses time knowledge taught according to the UK National Curriculum Statutory Guidance, and it is accompanied by practical resources such as worksheets to help teachers and other professionals address gaps in knowledge about time. The sections included in the assessment also overlap with those included in the Time Concept Questionnaire (Quartier, 2008) and the Time Knowledge Questionnaire (Labrell et al., 2016), which is indicative of content validity. However, in relation to construct validity, the scores from one section, Organisation of time, did not correlate with those from other sections. As mentioned previously there are a number of explanations for this which warrant further investigation.

In conclusion, while further research is needed to establish the reliability and validity of the Time Screening Assessment, the present study provides normative data which is the first step towards creating a standardized, norm-referenced assessment tool. The present study identified that two-thirds of the 79 pupils in this sample of 10 to 11-year olds had well-developed time related competencies, however seven pupils had not acquired the time knowledge that they would be expected to have at their age. As a result, they may later experience difficulties when talking about time and with organising their activities. Time-related skills are valuable as children get older, for example: when they are more likely to be responsible for getting themselves to school; where time concepts appear across different topics in the curriculum; where good time organisational skills are needed to complete the increasing amounts of homework on time and are associated with performing well in examinations. The Time 
Screening Assessment can be used by teachers and other professionals to identify children and young people with poor time knowledge so that they can receive targeted support.

\section{ORCID}

Nicola Brace (iD) https://orcid.org/0000-0003-2928-7327

Rosalind Herman (D) https://orcid.org/0000-0001-5732-9999

\section{REFERENCES}

Andersson, U. (2008). Mathematical competencies in children with different types of learning difficulties. Journal of Educational Psychology, 100, 48-66. http://dx.doi.org/10.1037/0022-0663.100.1.48.

Bryan, K., Freer, J. \& Furlong, C. (2007). Language and communication difficulties in juvenile offenders. International Journal of Language \& Communication Disorders, 42, 505-520. http://dx.doi.org/10.1080/13682820601053977.

Burny, E., Valcke, M. \& Desoete, A. (2009). Towards an agenda for studying learning and instruction focusing on time-related competences in children. Educational Studies, 35, 481-492. http://dx.doi.org/10.1080/03055690902879093.

Burny, E., Valcke, M. \& Desoete, A. (2012). Clock reading: An underestimated topic in children with mathematics difficulties. Journal of Learning Disabilities, 45, 351-360. http://dx.doi.org/10.1177/0022219411407773.

Doran, C., Dutt, S. \& Pembery, J. (2015). Time Matters: A Practical Resource to Develop Time Concepts and Self-Organisational Skills in Older Children and Young People. London: Speechmark.

Dutt, S. \& Doran, C. (2013). The meaning of time. Bulletin: the official magazine of the Royal College of Speech \& Language Therapists, February, 11.

Friedman, W.J. (1991). The development of children's memory for the time of past events. Child Development, 62, 139-155. http://dx.doi.org/10.2307/1130710.

Friedman, W.J. (1992). Children's time memory: The development of a differentiated past. Cognitive Development, 7, 171-187. http://dx.doi.org/10.1016/0885-2014(92)90010-O.

Friedman, W.J. (2005). Developmental and cognitive perspectives on humans' sense of the times of past and future events. Learning and Motivation, 36, 145-158. http://dx.doi.org/10.1016/j.lmot.2005.02.005.

Friedman, W.J. \& Laycock, F. (1989). Children's analog and digital clock knowledge. Child Development 60, 357-371. http://dx.doi.org/10.2307/1130982.

Hollmann, C.M. \& McNamara, J.R. (1999). Considerations in the use of active and passive parental consent procedures. The Journal of Psychology, 133, 141-156. http://dx.doi.org/10.1080/00223989909599729.

Labrell, F., Mikaeloff, Y., Perdry, H. \& Dellatolas, G. (2016). Time knowledge acquisition in children aged 6 to 11 years and its relationship with numerical skills. Journal of Experimental Child Psychology, 143, 1-13. http://dx.doi.org/10.1016/j.jecp.2015.10.005.

Quartier, V. (2008). Le développement de la temporalité: Théorie et instrument de mesure du temps notionnel chez l'enfant [Temporality development: Theory and instrument to measure notional time in children]. Approche Neuropsychologique des Apprentissages chez l'Enfant, 100, 76-85.

Quartier, V., Zimmermann, G. \& Nashat, S. (2010). Sense of time in children with AttentionDeficit/Hyperactivity Disorder (ADHD). Swiss Journal of Psychology, 69, 7-14.

Siegler, R.S. \& McGilly, K. (1989). Strategy choices in children's time-telling. In: I. Levin \& D. Zakay (Eds.) Time and human cognition: A life span perspective (pp.185-218). Amsterdam: Elsevier Science Publishers. 
Williams, D., Boucher, J., Lind, S. \& Jarrold, C. (2013). Time-based and event-based prospective memory in autism spectrum disorder: The roles of executive function and theory of mind, and time-estimation. Journal of Autism and Developmental Disorders, 43, 1555-1567. http://dx.doi.org/10.1007/s10803-012-1703-9. 